\title{
Trajectory Options for Human Mars Missions
}

\author{
Paul D. Wooster* \\ Massachusetts Institute of Technology, Cambridge, Massachusetts, 02139 \\ Robert D. Braun ${ }^{\dagger}$ \\ Georgia Institute of Technology, Atlanta, Georgia, 30332 \\ Jaemyung $\mathrm{Ahn}^{*}$ \\ Massachusetts Institute of Technology, Cambridge, Massachusetts, 02139 \\ and \\ Zachary R. Putnam ${ }^{\S}$ \\ Georgia Institute of Technology, Atlanta, Georgia, 30332
}

\begin{abstract}
This paper explores trajectory options for the human exploration of Mars, with an emphasis on conjunction-class missions. Conjunction-class missions are characterized by short in-space durations with long surface stays, as opposed to the long in-space durations and short surface stays characteristic of opposition-class missions. Earth-Mars and MarsEarth trajectories are presented across a series of mission opportunities and transfer times in order to explore the space of possible crew and cargo transfer trajectories. In the specific instance of crew transfer from Earth to Mars, the potential for aborting the mission without capture into Mars orbit is also of interest. As such two additional classes of trajectories are considered: free-return trajectories, where the trajectory would return the crew to Earth after a fixed period of time; and propulsive-abort trajectories, where the propulsive capability of the transfer vehicle is used to modify the trajectory during a Mars swing-by. The propulsive requirements of a trajectory, due to their associated impact on spacecraft mass, are clearly of interest in assessing trajectories for human Mars missions. Beyond the propulsive requirements, trajectory selection can have a significant impact on the entry velocity and therefore the aeroassist system requirements. The paper suggests potential constraints for entry velocities at Earth and Mars. Based upon Mars entry velocity, the 2year period free-return abort trajectory is shown to be less desirable than previously considered for many mission opportunities.
\end{abstract}

\section{Introduction}

$\mathrm{T}$ HIS paper provides an overview of interplanetary trajectory options for the human exploration of Mars, taking into account propulsive, crew support, and aeroassist requirements, with the intent of providing data useful for planning human missions to Mars. In order to provide a full range of information for mission planners, the trajectory options are examined across the 8 mission opportunities between 2020 and 2037, inclusively. As the complete period of the positions of the Earth and Mars in their orbits is approximately 15 years, the data can thus be employed for conceptual design of Mars missions for any mission opportunity. The primary metrics computed for the trajectory options include the propulsive delta-v required to escape the departure planet and reach the desired trajectory, the in-space transfer duration, and the entry velocity at the arrival planet. Each of these parameters is important for the design of spacecraft in terms of propulsion, crew habitation, and aeroassist systems. (In cases where propulsive as opposed to aerodynamic capture is used, the entry velocity can be converted to the required propulsive capture delta-v by subtracting the desired pericenter velocity of the captured orbit from the entry

\footnotetext{
* Research Assistant, Department of Aeronautics and Astronautics, 33-409, Member.

Associate Professor, School of Aerospace Engineering, 270 Ferst Drive, Associate Fellow.

\# Research Assistant, Department of Aeronautics and Astronautics, 33-409, Student Member.

${ }^{\S}$ Research Assistant, School of Aerospace Engineering, 270 Ferst Drive, Student Member.
} 
velocities provided.) In addition, the choice of trajectories dictates the available stay time at Mars which is also provided.

\section{A. Interplanetary Trajectory Modeling}

Two major types of human Mars missions have been proposed based upon their interplanetary trajectories and associated Mars stay times, known as conjunction-class (or long-stay) and opposition-class (or short-stay) missions. ${ }^{1}$ Conjunction-class missions are characterized by long stay times on Mars (order of 400 to 600 days), short in-space durations (approximately one year total for the Earth-Mars and Mars-Earth legs), and relatively small propulsive requirements. Opposition-class missions have significantly shorter Mars stay times (order of 30 to 90 days), long inspace durations (approximately 1.5 years total for the Earth-Mars and Mars-Earth legs), and relatively large propulsive requirements. As conjunction-class trajectories offer increased benefit (greater Mars stay time), at lower cost (propulsive requirements) and lower risk (due to the decreased time in deep-space), they serve as the focus for the trajectories in this paper.

The conjunction trajectories explored in this paper include both Earth-Mars and Mars-Earth legs in which the trip time is constrained to various durations between 180 and 270 days. This provides an overview of savings in trip time which can be gained through increases in propulsive delta-v, and can be useful for planning both crew and cargo transfers. An example of such trajectories is provided in Figure 1. In addition, for the crew transfer from Earth to Mars, the option to provide a free return abort capability may offer benefits. In such a transfer, the spacecraft is placed on a transfer from the Earth to Mars which also returns to Earth at a specified point in the future, without the need for any action by the spacecraft in order to enact such a transfer (in practical usage, mid-course corrections would likely be necessary). Typically such trajectories employ a resonance in the period between the transfer trajectory and the orbit of the Earth about the Sun. ${ }^{2}$ In this paper, free return trajectories with a 2 year period or 2:1 Earth to spacecraft revolution about the Sun resonance, and with a 1.5 year period, or 3:2 Earth to spacecraft revolution about the Sun resonance, are investigated.

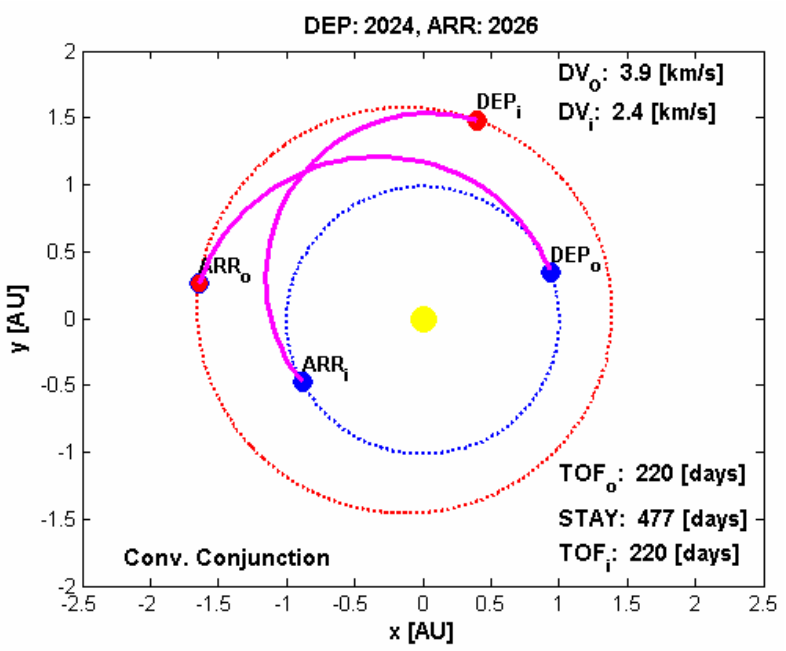

Figure 1. Example outbound (Earth-Mars) and inbound (Mars-Earth) conjunction trajectories relative to the orbits of the Earth and Mars for an Earth departure in 2024 and an Earth return in 2026. Both trajectories are fast conjunction transfers with transit durations of 220 days. The $x$ and $y$ axes are in Astronomical Units (AU).

The 2:1 resonant trajectories are called 2 year free return trajectories, as the period of time from Earth departure to Earth return will be 2 years if the abort option is taken. Similarly, the 3:2 resonant trajectories are called 3 year free return trajectories as the time from Earth departure to return is 3 years in the case of an abort. This paper also explores an option for a propulsive abort trajectory, in which if the crew opts to forgo capture at Mars, an abort burn is performed which places the spacecraft on an interplanetary trajectory that will return the crew to Earth. For the purposes of this paper, such trajectories have an Earth departure to Earth return time (in the case of an abort) of slightly over 2 years. Both the free return and the propulsive abort trajectories offer the prospect for the crew to return home without capture at Mars in the case of failure of some system already at Mars or otherwise required for the safe return of the crew to Earth. Both abort trajectory options clearly place a requirement on the outbound spacecraft of being able to support the crew during the lengthy return to Earth, and in the case of the propulsive abort trajectories add an additional critical event in the case of an abort. Whether such abort trajectories are employed is a decision to be analyzed by mission planners and eventually determined by programmatic decision makers. The information in this paper is intended to serve as a reference in assisting with such decisions.

The interplanetary trajectories examined in this paper make use of the J2000 ephemeredes for determining planetary positions. ${ }^{3}$ The delta-v's are assumed to be impulsive in nature. A patched conic method with the 
assumption that the sphere of influence radii of planets are negligibly small relative to the interplanetary path lengths traveled is used for the trajectory calculation. ${ }^{4}$ All trajectories (conventional inbound / outbound, free return, and propulsive abort) were optimized for minimum departure $\mathrm{C} 3$ energy (and thus minimum departure delta-V). In more detailed trajectory design, attempts to also optimize other parameters such as entry velocity would likely be worthwhile. Sequential quadratic programming (SQP) is used for the optimization. All interplanetary trajectory code was written and executed in MATLAB.

\section{B. Aeroassist Modeling}

Aeroassist maneuvers will likely be required for human Mars exploration to decrease vehicle mass. Aeroassist maneuvers performed at both Mars arrival and Earth return virtually eliminate the propellant required to slow mission assets down in an all-propulsive architecture. Previous analyses estimate that the use of aeroassist maneuvers may offer significant reductions in the initial mass of a crewed Mars vehicle, potentially by greater than $50 \%$. ${ }^{5,6}$ This reduction in vehicle mass implies a similar reduction in cost which may be mission enabling.

Aeroassist trajectories use a planetary atmosphere to alter the vehicle velocity vector non-propulsively. Through aeroassist, the vehicle velocity vector magnitude and direction may be altered through judicious use of aerodynamic forces. Aeroassist trajectories dissipate vehicle energy through heat transfer, creating an extreme thermal environment which a successful entry vehicle must survive. The peak heat rate, integrated heat load, and deceleration environment experienced by an entry vehicle are functions of vehicle mass, aerodynamics, aeromaneuvering, and the vehicle state at atmospheric interface. High speed steep entries incur the highest heat rates and largest peak decelerations, while high speed shallow entries incur the largest integrated heat loads. In all cases, the aerothermal environment becomes more extreme with increasing entry velocity magnitude. However, an entry vehicle may utilize aeromaneuvering to minimize the severity of the aerothermal and deceleration environments. Two types of aeroassist maneuvers are relevant to human Mars missions: entry and aerocapture.

Entry maneuvers use a planetary atmosphere to decelerate from high velocity and descend to the planet's surface (Figure 2). Entry may occur from inbound hyperbolic trajectories or from orbit. Entry from orbit requires a small propulsive maneuver to lower the periapsis of the vehicle orbit into the atmosphere.

Aerocapture maneuvers use a planetary atmosphere to decelerate from a hyperbolic trajectory to a bound orbit during a single atmospheric pass. Aerodynamic drag incurred during the atmospheric pass reduces the vehicle's energy to a desired value. After the vehicle exits the atmosphere with the correct energy, a propulsive maneuver is required to raise the periapsis of the vehicle's orbit out of the atmosphere.

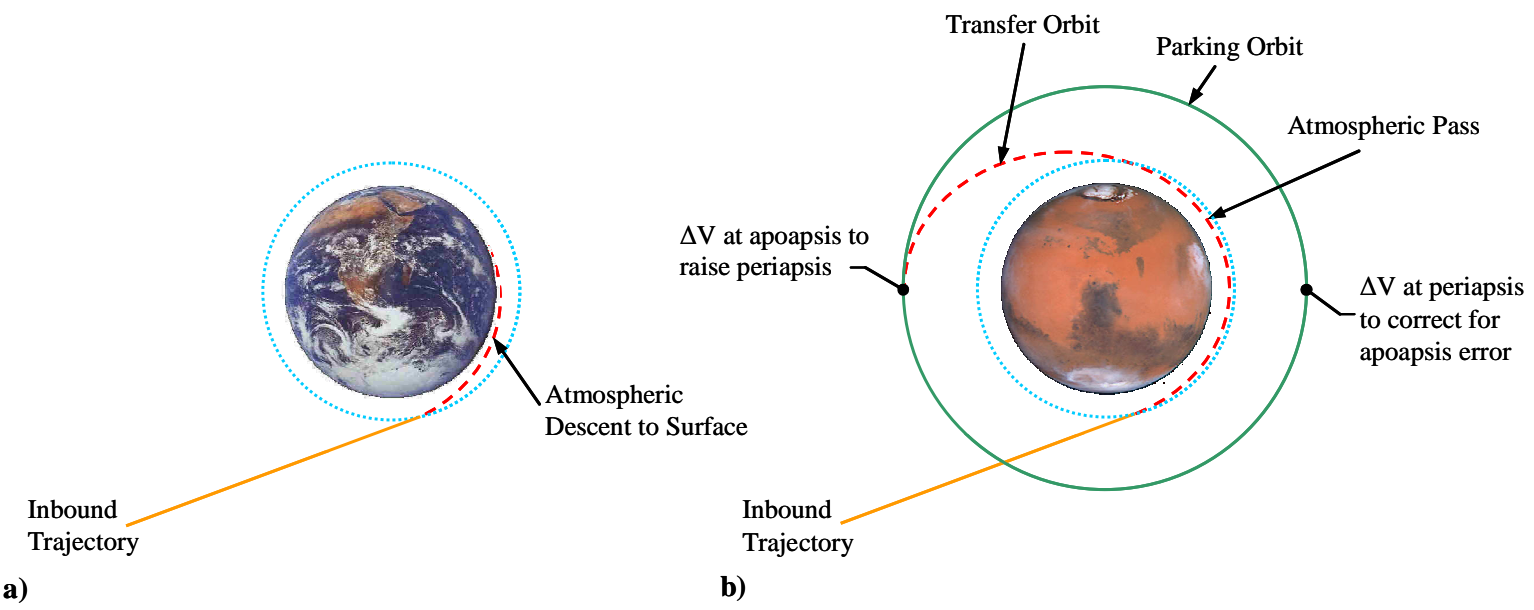

Figure 2. Human Mars mission aeroassist trajectories: (a) entry and (b) aerocapture.

Entry trajectory analysis of aeroassist maneuvers at Earth and Mars was performed with the three-degree-offreedom version of the Program to Optimize Simulated Trajectories (POST). ${ }^{7}$ The linear feedback control option 
was used to fly constant altitude and constant deceleration entry trajectories by utilizing lift control through bank angle modulation. Historic aerodynamic data was used when available for this analysis. For non-heritage vehicles, aerodynamic analysis was performed using the tangent cone method option in the Aerodynamic Preliminary Analysis System (APAS) ${ }^{8}$ The fidelity of the aerodynamic data in the hypersonic regime generated by APAS is on the order of the fidelity of the entry trajectory analyses performed. Aeroheating calculations were performed with two stagnation point heating approximation methods. Convective heating was calculated using Chapman's equation ${ }^{9}$ and radiative heating was calculated using the Tauber-Sutton approximation ${ }^{10}$. The results for both calculations were summed to find the total stagnation point heat rate and integrated heat load as a function of time for each trajectory.

\section{Standard Conjunction Trajectory Options for Earth-Mars and Mars-Earth Transfers}

In this section we explore fast conjunction trajectory options for Earth-Mars and Mars-Earth transfers with transfer durations set in 10 day increments from 180 to 270 days. 180 days represents a suggested fast conjunction transfer time from previous studies ${ }^{5,11,12} ; 270$ days represents the maximum Type I (less than 180 degree transfer about the Sun) transfer duration. The values of departure delta-v and arrival entry velocity are presented for each of the trajectories. In addition, the Mars stay times associated with the outbound (Earth-Mars) transits are presented relative to a fixed duration inbound (Mars-Earth) return transfer, followed by the sensitivity of Mars stay time to the inbound transfer duration.

\section{A. Earth-Mars Outbound Trajectories}

Figure 3 shows the Earth departure delta-v for fast conjunction transfers with durations of 180 to 270 days. These trajectories are suitable for cargo flights as well as crew flights (if an abort option is not selected) to Mars. In observing the delta-v graph, one can see that in any particular departure opportunity a trade exists between the delta$\mathrm{v}$ and the flight time, although the minimum delta-v only rarely corresponds with the maximum transfer time. It is useful to consider the delta-v required to be able to make an Earth-Mars transfer in any opportunity, as this could be used in sizing a system which could then operate across all opportunities, supporting a continuous sequence of Mars missions. In this case the delta-v required if transit time is not an issue is just under $4 \mathrm{~km} / \mathrm{s}(3.98 \mathrm{~km} / \mathrm{s}$ in our analysis). Interestingly, this is very close to the delta-v required to enable a 180 day transfer in every opportunity $(4.08 \mathrm{~km} / \mathrm{s}$ in our analysis). As such, it does not appear that enabling 180 day (or faster) Earth-Mars transfers in all mission opportunities will be overly burdensome from a delta-v perspective.

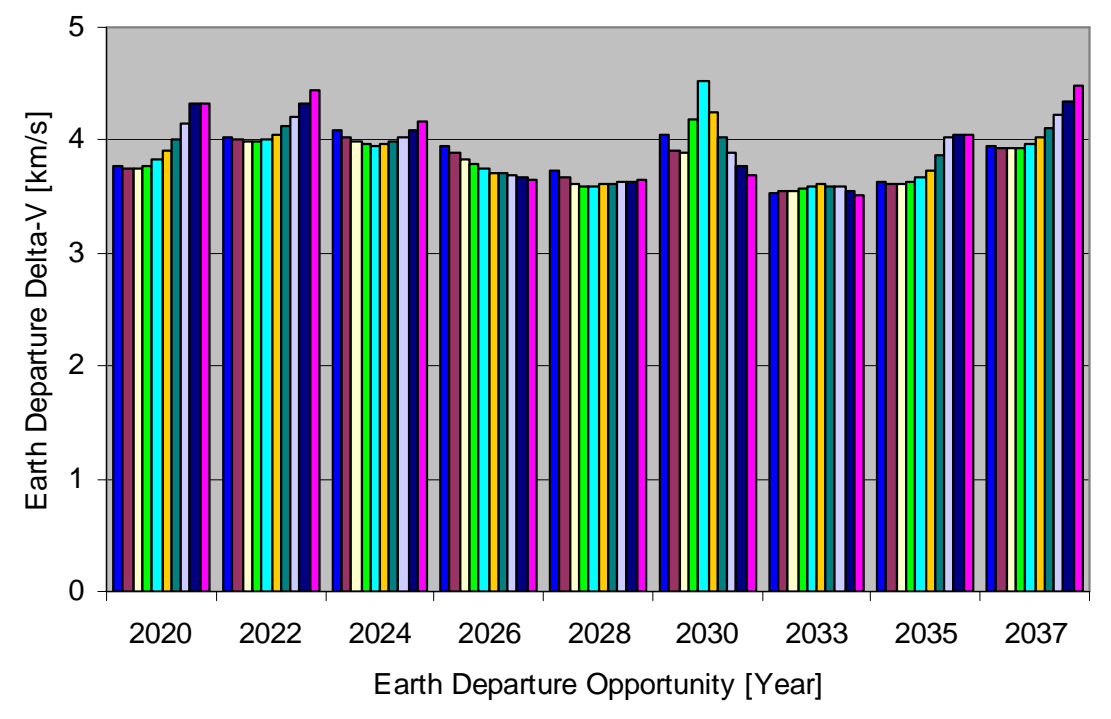

- Fast Conjunction (180 day outbound) $\square$ Fast Conjunction (190 day outbound) 口 Fast Conjunction (200 day outbound) 口 Fast Conjunction (210 day outbound) $\square$ Fast Conjunction (220 day outbound) $\square$ Fast Conjunction (230 day outbound) $\square$ Fast Conjunction (240 day outbound) 口 Fast Conjunction (250 day outbound) - Fast Conjunction (260 day outbound) $\square$ Fast Conjunction (270 day outbound)

Figure 3. Low Earth orbit departure Delta-V for Earth-Mars fast-conjunction trajectories with transit times from 180 to 270 days, over Earth departure opportunities from 2020 to 2037. 
Figure 4 shows the Mars arrival entry velocity for the same set of trajectories. In contrast to the departure delta$v$ 's, the arrival entry velocities tend to show a stronger correlation between lower entry velocities and longer transit times, in particular in the cases with higher entry velocities. The minimum required entry velocity capability to support missions across all opportunities is approximately $7.26 \mathrm{~km} / \mathrm{s}$, driven by the shallow minimum in the 2028 mission opportunity, and enabling a 210 day transfer in that case. If the Mars aerocapture/entry system were developed so as to support missions in all opportunities (meaning entry velocities of up to $7.26 \mathrm{~km} / \mathrm{s}$ ), the 2030 opportunity would end up with the longest transit time, namely 240 days, although in many cases transit times of 180 days (or less) would be feasible with such a system. The 2030 opportunity in general appears to offer the greatest challenge for faster transits from an entry velocity perspective, requiring a $9.62 \mathrm{~km} / \mathrm{s}$ entry velocity for a 180 day transfer and an $8.27 \mathrm{~km} / \mathrm{s}$ entry velocity for a 200 day transfer. (It should also be noted that for the 2030 case, while increasing the transit time further does decrease the entry velocity, doing so increases the Earth departure delta-v significantly above $4 \mathrm{~km} / \mathrm{s}$ until transit times of approximately 240 days are reached.)

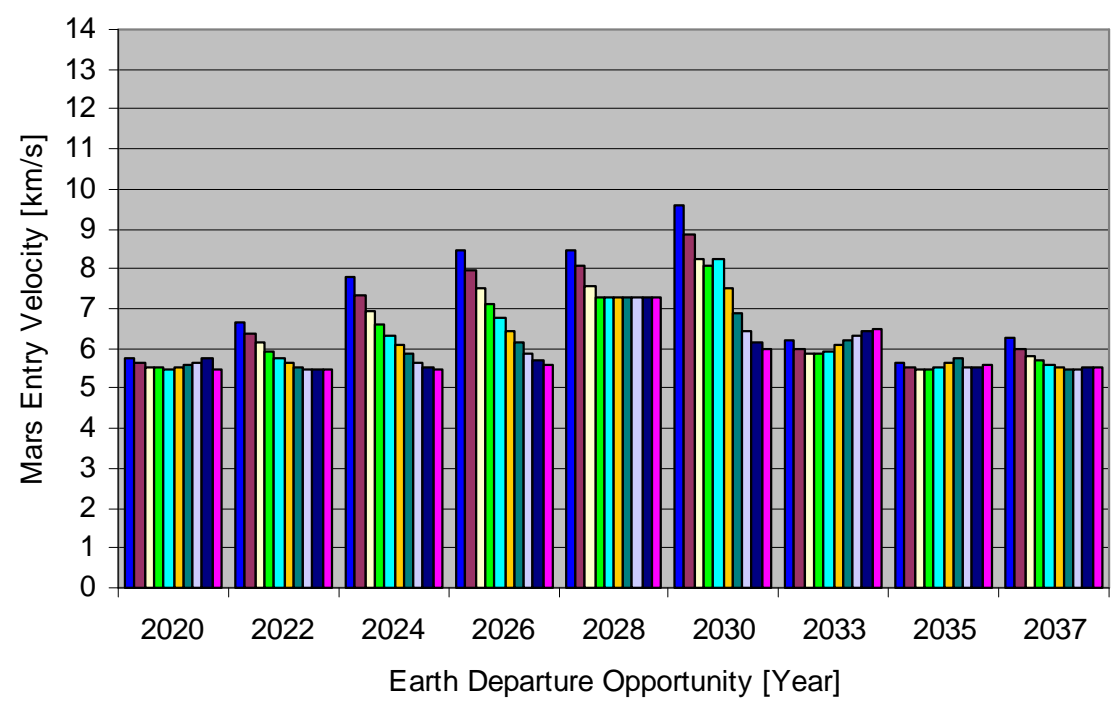

\begin{tabular}{|l|}
\hline Fast Conjunction (180 day outbound) \\
$\square$ Fast Conjunction (190 day outbound) \\
$\square$ Fast Conjunction (200 day outbound) \\
$\square$ Fast Conjunction (210 day outbound) \\
$\square$ Fast Conjunction (220 day outbound) \\
$\square$ Fast Conjunction (230 day outbound) \\
$\square$ Fast Conjunction (240 day outbound) \\
$\square$ Fast Conjunction (250 day outbound) \\
Fast Conjunction (260 day outbound) \\
$\square$ Fast Conjunction (270 day outbound)
\end{tabular}

Figure 4. Mars arrival entry velocities for Earth-Mars fast-conjunction trajectories with transit times from 180 to 270 days, over Earth departure opportunities from 2020 to 2037.

In viewing the Mars entry velocity and Earth departure delta-v information together, it appears that Mars entry velocity will be a stronger constraint on transit times than Earth departure delta-v. Given that cargo missions are likely to be insensitive to transit times, it appears that developing cargo transportation systems such that they can provide an Earth departure delta-v of approximately $4 \mathrm{~km} / \mathrm{s}$ and support Mars entry velocities of up to $7.26 \mathrm{~km} / \mathrm{s}$ (both with appropriate margin) would be reasonable. For crew missions utilizing standard fast conjunction trajectories, consideration of increasing the Mars entry velocity capability may be worthwhile in order to enable faster Earth-Mars transit times in the more challenging opportunities.

\section{B. Mars-Earth Inbound Trajectories}

Figure 5 shows the Mars departure (Trans-Earth Injection) delta-v for each Earth return opportunity from 2020 to 2037 for transfers with return transit durations of 180 to 270 days, based upon return from a low Mars orbit ${ }^{*}$. It should be noted that due to the nature of the arrival and departure dates, a crew transiting to Mars in one Earth

\footnotetext{
* Options to decrease the required Earth return delta-v exist through the use of an elliptic Mars rendezvous orbit, in the case of architectures involving a Mars orbit rendezvous prior to return. Such elliptic orbits effectively transfer a portion of the Earth return delta-v from the Earth return vehicle towards the Mars ascent vehicle. The quantity of delta-v to be transferred is subject to trade (along with other complexities arising from the use of elliptic orbits), and if such a strategy is used the transferred delta-v can be subtracted from these figures. The maximum delta-v that can be transferred is approximately $1.3 \mathrm{~km} / \mathrm{s}$, in order to keep the Earth return vehicle in a bound Mars orbit with reasonable orbital period.
} 
departure opportunity would first be able to return to Earth in the next Earth return opportunity. For example, a crew transiting from Earth to Mars in the 2024 Earth departure opportunity would be able to return in the 2026 Earth return opportunity (or any subsequent one, in the case of longer duration stays). In analyzing the data, again a trade is found to exist in any given opportunity between delta- $\mathrm{v}$ and transit duration, with the minimum delta- $\mathrm{v}$ coming about through increased trip times in some but not all opportunities. If any transit duration between 180 and 270 days is allowed, an Earth return delta-v of $2.6 \mathrm{~km} / \mathrm{s}$ is required, which is driven by the 2037 opportunity and enables a 260 day transit in that case. Increasing the delta-v slightly to $2.7 \mathrm{~km} / \mathrm{s}$ reduces the maximum transit time across all opportunities to 200 days, a significant reduction, and would enable faster transfers in a number of opportunities. Further increasing the delta-v to $3 \mathrm{~km} / \mathrm{s}$ allows for transit times of 180 days or less in all opportunities.

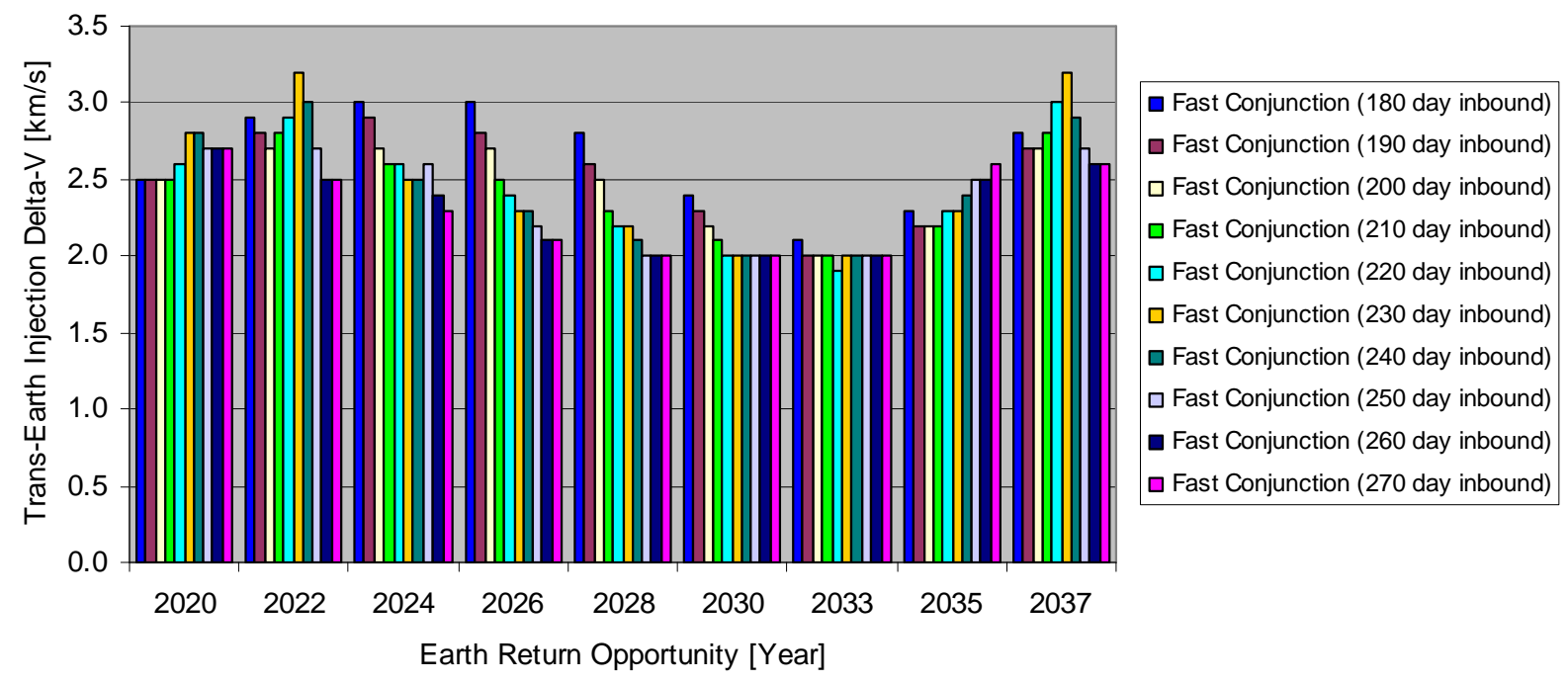

Figure 5. Low Mars orbit departure Delta-V for Mars-Earth fast-conjunction trajectories with transit times from 180 to 270 days, over Earth return (Mars departure) opportunities from 2020 to 2037.
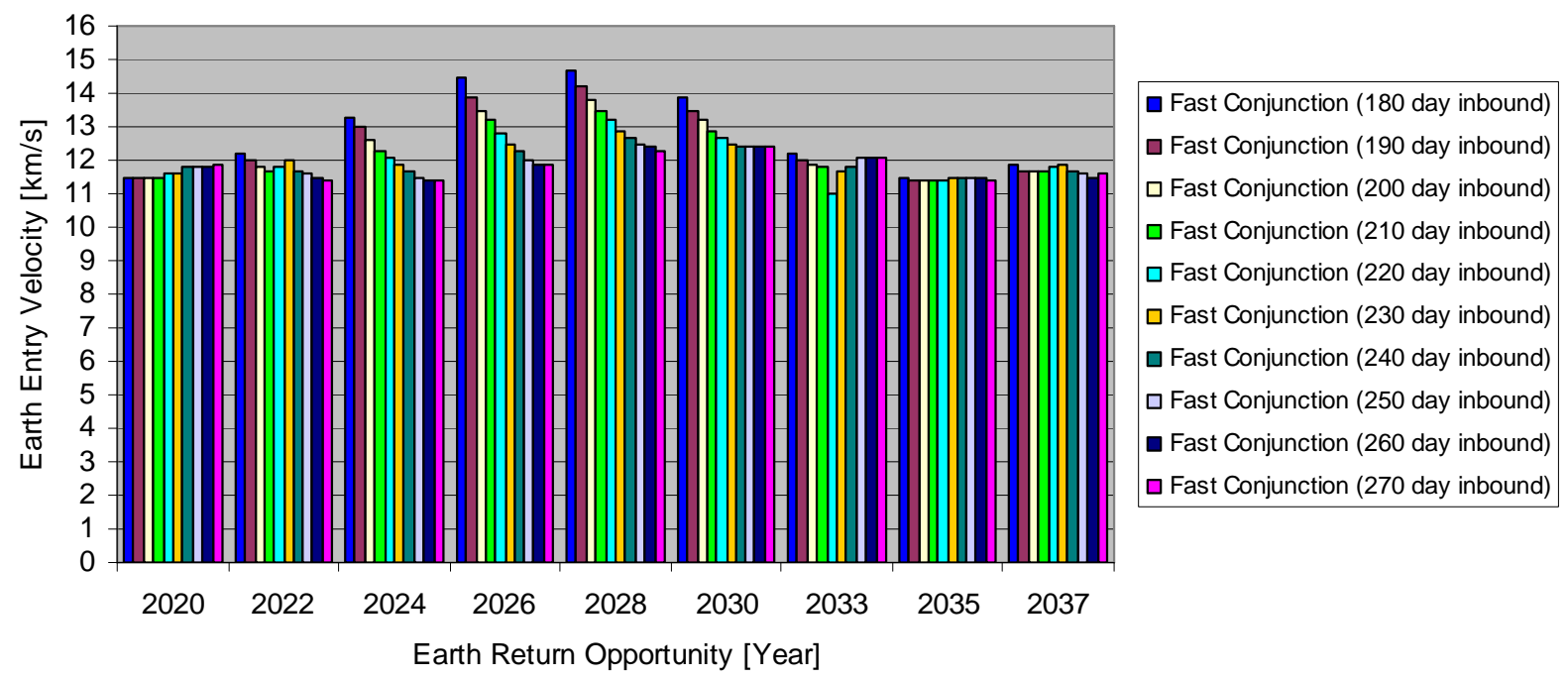

Figure 6. Earth arrival entry velocities for Mars-Earth fast-conjunction trajectories with transit times from 180 to 270 days, over Earth return (Mars departure) opportunities from 2020 to 2037.

The Earth entry velocities for the same set of Mars-Earth transfers are shown in Figure 6. Here again a stronger correlation is found between decreased transit times and increased Earth entry velocities, particularly in the 
opportunities featuring higher entry velocities. An Earth entry velocity capability of $12.4 \mathrm{~km} / \mathrm{s}$ is sufficient to enable Earth return in any opportunity if transit duration is not a constraint. As the Mars-Earth transit is being used by the crew, decreasing transit time is highly desirable. In order to return in 180 days, an Earth entry velocity capability of $14.7 \mathrm{~km} / \mathrm{s}$ would be required (driven by the 2028 opportunity). If an Earth entry limit of $14 \mathrm{~km} / \mathrm{s}$ were imposed, the 2028 opportunity would be constrained to a 200 day return transfer and 2026 would be constrained to a 190 day return, with the remainder of opportunities able to support 180 days or less. Further reducing the Earth entry velocity capability to $13 \mathrm{~km} / \mathrm{s}$ would require a 230 day return in 2028, a 220 day return in 2026, a 210 day return in 2030, a 190 day return in 2024, and enable returns of 180 days or less in the remaining opportunities. Clearly a trade thus exists between the entry velocity capability of the Earth entry vehicle (along with the technology development associated to achieve this) and the requirements associated with supporting the crew for somewhat longer durations.

\section{Mars Stay Time Associated with Conjunction Transfers}

In addition to impacting the in-space duration, propulsive, and entry velocity requirements, the choice of Mars mission trajectories will also determine the stay time available at Mars. The stay time available will impact both the quantity of exploration that can be performed and the systems required to support the crew while at Mars. Figure 7 provides information on the Mars stay time resulting from the choice of outbound Earth-Mars trajectories. The values are provided relative to an Earth return transfer of 270 days in the immediately following return opportunity (i.e., the earliest return opportunity available). Figure 8 shows the change in stay time of various return transfers relative to the 270 day transfer presented in Figure 7. It is interesting to note that there can be significant variation in stay time from opportunity to opportunity and within an opportunity based upon outbound transit time, with stay times of just over 400 days on the low end and just over 600 days on the high end. It tends to be that for the most part faster Earth-Mars transit times result in longer Mars stay times, although this trend is stronger in some opportunities than in others. In the case of return, decreasing the Mars-Earth transit time can either increase or decrease Mars stay time, although the sensitivity of stay time to Mars-Earth transit time appears to be lower than to Earth-Mars transit time. While we believe it is unlikely that surface duration considerations will be a major driver in trajectory option selection (within the family of conjunction-class trajectories), including considerations of the increased benefit of longer surface durations in trajectory trades could be worthwhile, in particular in terms of the change in stay time with varying Earth-Mars transit times.

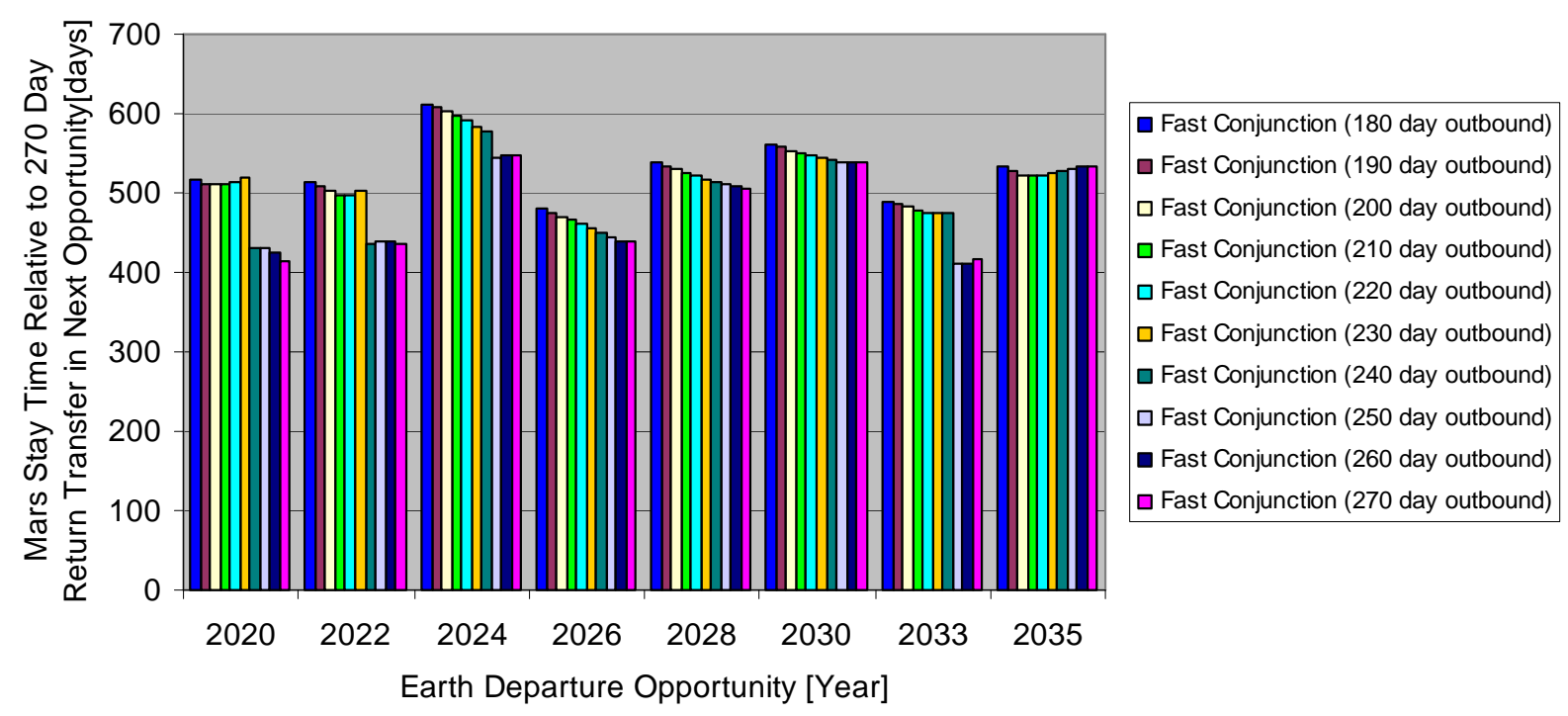

Figure 7. Mars stay times for Earth-Mars fast-conjunction trajectories with transit times from 180 to 270 days, over Earth departure opportunities from 2020 to 2035. The stay times are computed assuming a MarsEarth return transfer of 270 days in the immediately following Earth return opportunity (e.g., the 2022 Earth return opportunity in the case of a 2020 Earth departure). 


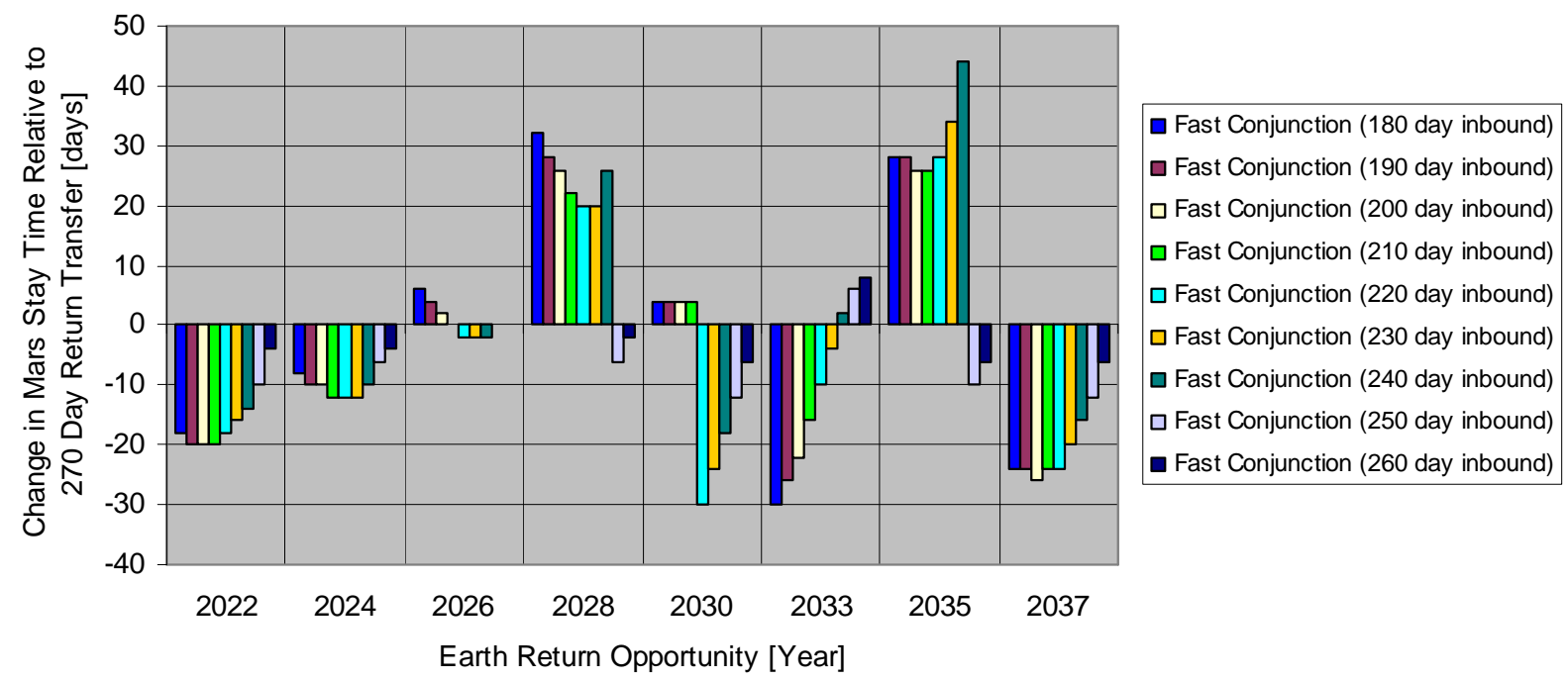

Figure 8. Change in Mars stay time for Mars-Earth fast-conjunction trajectories with transit times from 180 to 260 days relative to a 270 day Mars-Earth transfer, over Earth return (Mars departure) opportunities from 2022 to 2037. Negative numbers represent a decrease in Mars stay time, positive numbers an increase.

\section{Earth-Mars Transfer Trajectory Abort Options}

The trajectories options discussed in the previous section could be used for either cargo or crew transfers to and from Mars. In the specific case of transfer to Mars, it may be desirable to provide an option to allow the crew to abort the mission without requiring capture into Mars orbit. Whether and how the need would arise to conduct such an abort would depend greatly upon the design of the human Mars exploration architecture, including the degree to which other contingency options exist. Examples that could potentially lead to such an abort include the failure of prepositioned systems required to either support the crew at Mars or return them to Earth, or systems on-board their outbound craft that are required in order to successfully capture into Mars orbit and/or possibly descend to the surface. It should be noted that the abort trajectory options presented here by no means provide a rapid return to Earth option - the total time spent in the aborting spacecraft can approach or even exceed the total duration of a nominal mission. As such, and again depending upon the Mars architecture employed, including the option for one of these aborts may have a significant impact upon the design of the outbound spacecraft. In addition, the abort option does not provide a mitigation for failure of crew support systems onboard the outbound spacecraft; in such a case it may be most advantageous to arrive at Mars as planned such that the crew can make use of other assets and resources present there. ${ }^{5,11,12}$

The abort trajectory options investigated in this paper include free return trajectories with orbital periods of 2 years and 1.5 years, and propulsive abort trajectories covering a range of impulsive abort delta-v's during a swingby at Mars. Other abort options, including free return trajectories with other periods (such as 1 or 3 years) or combined swing-bys of Mars and Venus have been proposed, however these are not included as they tend to have some combination of higher propulsive and entry velocity requirements, longer in-space transit times, and infrequent mission opportunities (in the case of abort trajectories involving Venus swing-bys). ${ }^{13}$

The free-return trajectories employ the periodicity inherent in the Earth's orbit around the Sun to return to the point in space where they departed from Earth at the same time that the Earth returns to that point in space. In the case of the 2 year free return investigated in this paper, the outbound spacecraft is on a 2 year period orbit, such that the spacecraft completes one orbit about the Sun (if arrival at Mars is aborted), while the Earth completes two revolutions about the Sun (see Figure 9). This free return represents the shortest Earth return time of any of the practical Earth-spacecraft resonant period free return trajectory options. Although a one year period orbit is theoretically possible, this requires the perihelion of the transfer orbit to be significantly inside the orbit of the Earth, 
resulting in a large angle between the Earth departure heliocentric velocity of spacecraft and the heliocentric velocity of the Earth, meaning a very large Earth departure delta- $v$ is required. For the three year free return option presented, the spacecraft is placed on a 1.5 year period heliocentric orbit for transfer to Mars, and in the case of the abort makes two complete orbits about the Sun while the Earth makes three revolutions, with the trajectory thus being called a 3 year free return. The lower energy of the transfer orbit makes this the lowest Earth departure delta-v option in a number of opportunities, although at the expense of increasing the transfer time to Mars and the time required to be spent in space in the case of an abort. While free return trajectory options with longer orbit periods exist (3 year period, 4 year period, etc.), they exhibit high Earth departure delta-v's due to their higher energy transfer orbits without offering any benefits in terms of time in-space during an abort.

In investigating the free return options outlined above, we discovered that although the 2 year free return trajectories were quite attractive in terms of in space duration, they suffered from high Mars entry velocities in a number of opportunities. As such, we decided to investigate the potential to reduce these Mars entry velocities through decreasing the initial transfer orbit energy somewhat and then employing a propulsive maneuver at Mars (in case of an abort) to transition to a trajectory that will return the crew to Earth, with a total in-space time similar to that of a 2 year free return trajectory (see Figure 10). In addition to decreasing the Mars entry velocity, such propulsive abort maneuvers were found to offer the additional benefit of decreasing the Earth departure delta-v. In order to decrease the impact upon the outbound spacecraft of including this option, we suggest that propulsion systems already onboard the spacecraft for maneuvers within the nominal mission (such as Mars landing or Earth return propulsion), be employed to perform the abort. As such, we investigated a range of abort delta-v's from $600 \mathrm{~m} / \mathrm{s}$, representative of the low end of delta-v required of Mars landing systems ${ }^{5}$, up to $2,700 \mathrm{~m} / \mathrm{s}$ which appears to be towards the upper end of anticipated trans-Earth injection delta-v's assuming a circular departure orbit as discussed in the previous section.

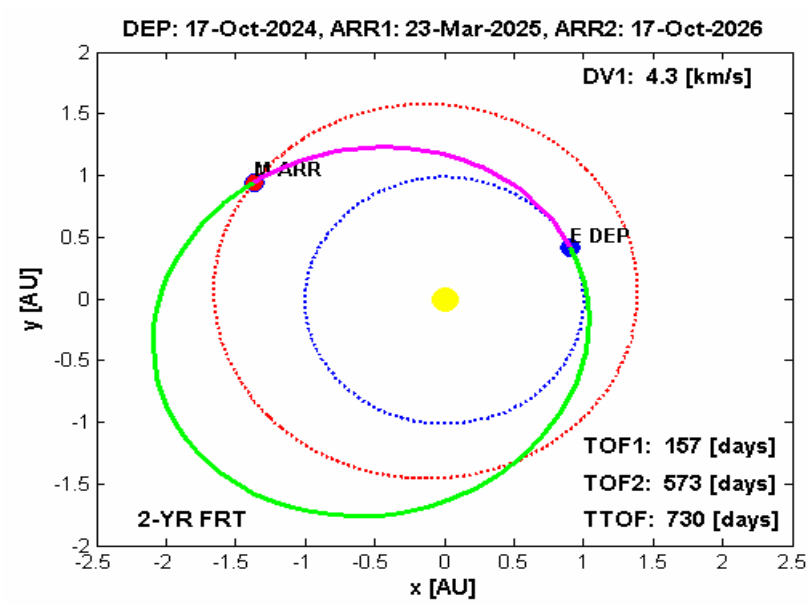

Figure 9. Example 2 year free return trajectory. The outbound spacecraft starts on an Earth-Mars transfer with a total orbital period of 2 years (magenta), and continues along that same trajectory after by-passing Mars in the case of an abort (green).

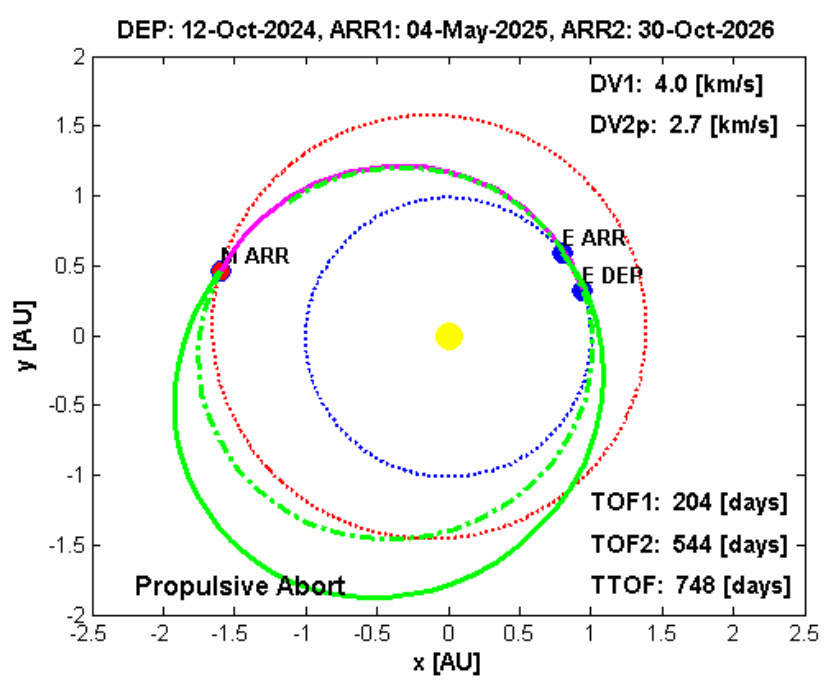

Figure 10. Example propulsive abort trajectory (with $2.7 \mathrm{~km} / \mathrm{s}$ abort delta-v). The outbound spacecraft starts on an Earth-Mars transfer (magenta), which if no action were taken would not return to Earth (dashed green). In the case of an abort a propulsive Mars swing-by maneuver shifts the trajectory to one that does arrive at Earth (solid green).

Figure 11 presents the delta-v required for departure from Earth for the free return and propulsive abort trajectory options investigated. The 2 year free return trajectory very consistently has an Earth departure delta-v of approximately $4.3 \mathrm{~km} / \mathrm{s}$ across all opportunities (ranging from 4.27 to $4.35 \mathrm{~km} / \mathrm{s}$ ). While there is greater variability from opportunity to opportunity, the propulsive abort options consistently provide a monotonic decrease in Earth departure delta-v with increasing abort delta-v, approaching the 2 year free return trajectory in the limit as the abort delta-v approaches zero. The 3 year free return (1.5 year period heliocentric orbit) trajectory exhibits the unique pattern of featuring a relatively low delta-v in many opportunities, and then a significantly higher delta-v in some opportunities (most notably 2022 and 2037, and to a lesser extent 2035). This behavior is because in some 
opportunities a 1.5 year period orbit must have its perihelion inside the orbit of the Earth in order to travel far enough out to reach Mars, resulting in increased angles between the desired heliocentric velocity of the orbit and the Earth's heliocentric velocity, and consequently increased Earth departure delta-v. In short, the 1.5 year period orbit is far away from the minimum energy Mars transfer in those opportunities. It is interesting to note that, with the exception of the 3 year free return at its peaks, all of these delta-v's are quite similar to the fast conjunction transfer trajectory options presented previously (less than a 10\% increase in the case of 2 year free returns), as such Earth departure propulsive considerations are unlikely to preclude the use of these abort trajectories.

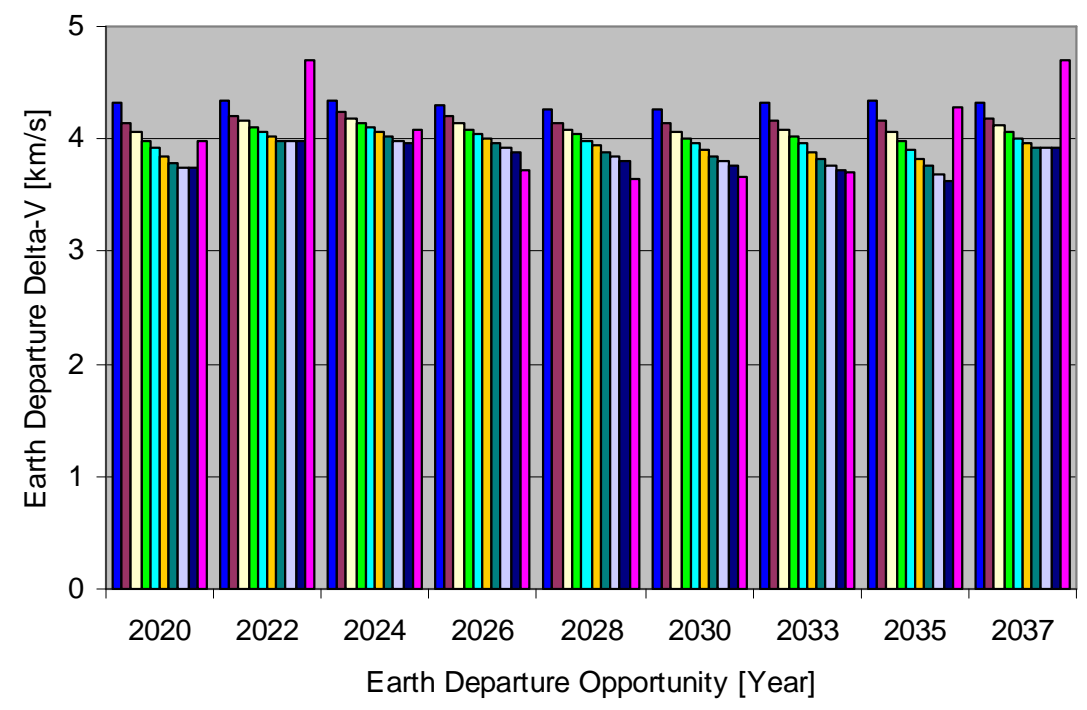

- Free Return (2 year return)

口 Propulsive Abort (0.6 km/s abort delta-v) 口 Propulsive Abort $(0.9 \mathrm{~km} / \mathrm{s}$ abort delta-v) $\square$ Propulsive Abort (1.2 km/s abort delta- $v$ ) $\square$ Propulsive Abort (1.5 km/s abort delta-v) $\square$ Propulsive Abort (1.8 km/s abort delta- $v$ ) $\square$ Propulsive Abort $(2.1 \mathrm{~km} / \mathrm{s}$ abort delta-v) $\square$ Propulsive Abort (2.4 km/s abort delta-v) - Propulsive Abort (2.7 km/s abort delta-v) $\square$ Free Return (3 year return)

Figure 11. Low Earth orbit departure Delta-V for Earth-Mars free return and propulsive abort trajectories over Earth departure opportunities from 2020 to 2037.

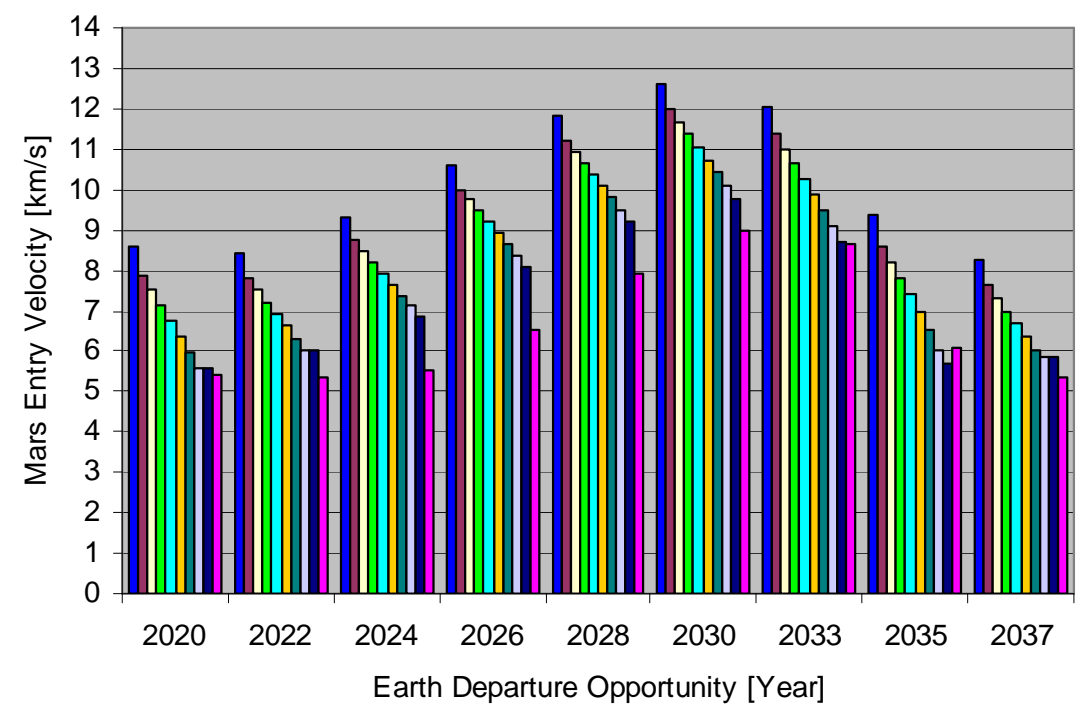

- Free Return (2 year return)

$\square$ Propulsive Abort (0.6 km/s abort delta-v) $\square$ Propulsive Abort $(0.9 \mathrm{~km} / \mathrm{s}$ abort delta-v) $\square$ Propulsive Abort (1.2 km/s abort delta-v) $\square$ Propulsive Abort (1.5 km/s abort delta-v) $\square$ Propulsive Abort (1.8 km/s abort delta-v) 口 Propulsive Abort $(2.1 \mathrm{~km} / \mathrm{s}$ abort delta-v) $\square$ Propulsive Abort $(2.4 \mathrm{~km} / \mathrm{s}$ abort delta- $\mathrm{v})$ - Propulsive Abort (2.7 km/s abort delta-v) $\square$ Free Return (3 year return)

Figure 12. Mars arrival entry velocities for Earth-Mars free return and propulsive abort trajectories over Earth departure opportunities from 2020 to 2037.

The Mars entry velocities of these trajectories are presented in Figure 12. Here there is a pronounced variation in entry velocity from opportunity to opportunity, and the entry velocities themselves can become quite high. The 2 year free return has the highest entry velocity, peaking over $12 \mathrm{~km} / \mathrm{s}$ in 2030 and 2033, and staying above $9 \mathrm{~km} / \mathrm{s}$ for all of the opportunities from 2024 to 2033, inclusive. As will be discussed in the following section, such entry velocities present a difficult challenge for Mars aerocapture systems, and may thus preclude the use of the 2 year 
free return in a number of mission opportunities. Including a propulsive abort capability can lower the Mars entry velocities significantly. By including an abort delta-v of $2.7 \mathrm{~km} / \mathrm{s}$ (consistent with the nominally trans-Earth injection delta-v capability of a vehicle designed to bring the crew back from low Mars orbit at the end of a mission), the delta-v can be kept below $10 \mathrm{~km} / \mathrm{s}$ in all mission opportunities. Utilizing instead an abort delta-v of 1.8 $\mathrm{km} / \mathrm{s}$ (consistent with an Earth return vehicle departing a highly elliptic Mars orbit), the entry velocity will peak at $10.7 \mathrm{~km} / \mathrm{s}$ in 2033, and be below $9 \mathrm{~km} / \mathrm{s}$ other than in the 2028, 2030, and 2033 opportunities. From an entry velocity perspective the 3 year free return option provides a distinct advantage in that it can remain below $9 \mathrm{~km} / \mathrm{s}$ in all opportunities.

Unlike the fixed transfer durations of the trajectories in the previous section, the Earth-Mars transit times for the abort trajectories vary based upon the opportunity as presented in Figure 13. For the most part, these trajectories offer comparable or faster transit times to the trajectories analyzed in the previous sections, with the exception of the 3 year free return. The 3 year free return exhibits close to a factor of two variation in transit time, from a minimum of 137 days in 2033 to a maximum of 262 days in 2035. While such transfer durations are not infeasible, keeping the outbound transfer durations below those exhibited by the 3 year free return in its longer transfers would be desirable.

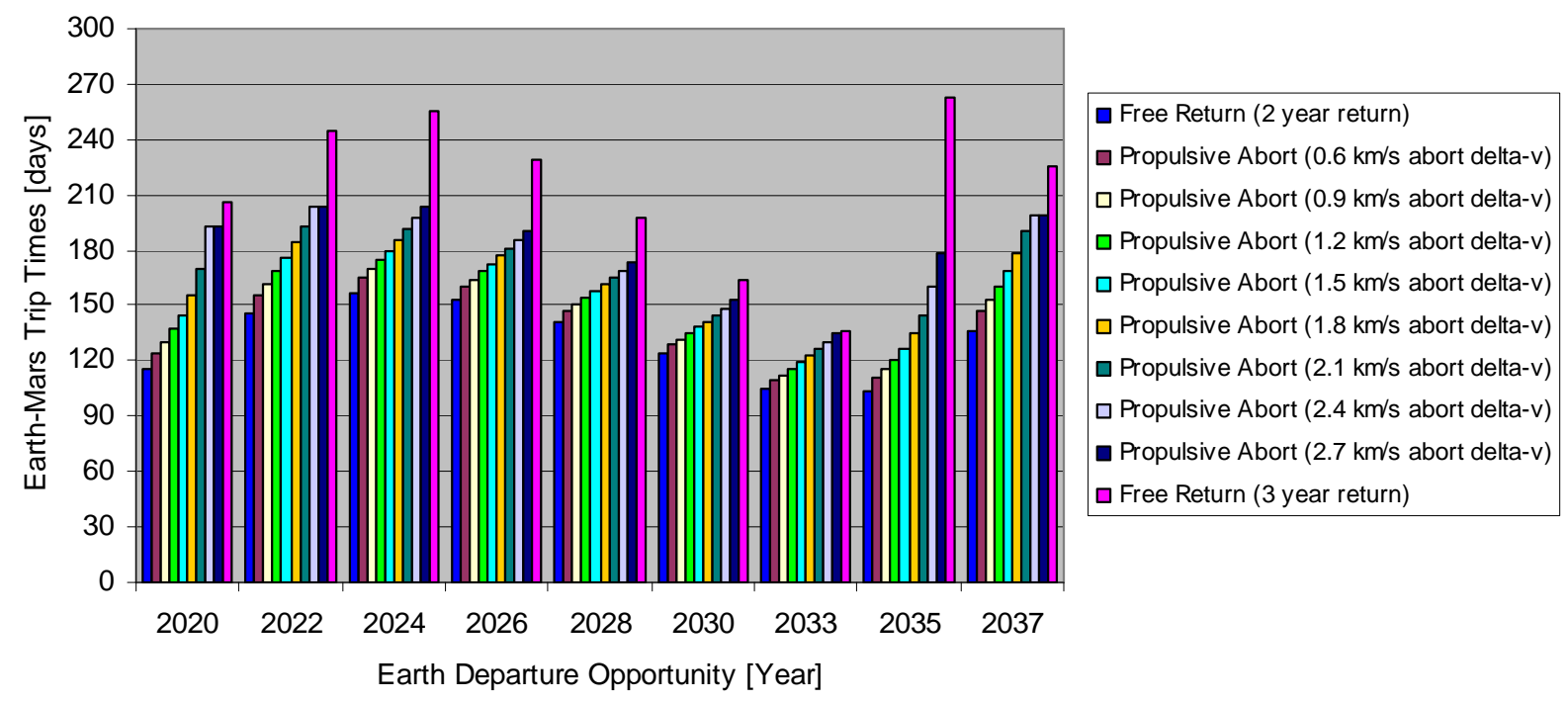

Figure 13. Earth-Mars transit times for Earth-Mars free return and propulsive abort trajectories over Earth departure opportunities from 2020 to 2037.

Beyond the considerations of transit to Mars, abort trajectories by their very nature must also consider their Earth return leg in case an abort is opted for. The Earth entry velocities, presented in Figure 14, are relatively benign, when considering that the peak entry velocities of approximately $12.5 \mathrm{~km} / \mathrm{s}$ are in line with the minimum entry velocity capability required for ensuring Mars return in all opportunities (without constraints on Mars-Earth transit time). As such, the next item to consider is the requirement to support the crew during the duration of their transit to Earth. Figure 15 presents the total in-space time in the case of an abort (this is the total time from Earth departure to Earth return, as this value will size the crew support requirements of the outbound spacecraft in order to support aborts). What can be seen is that the propulsive aborts all have very similar in-space durations to that of the 2 year free return, increasing marginally with increasing abort delta-v. The 3 year free return of course represents a 50\% increase in abort time over the 2 year free return. In terms of these durations, two years is of approximately the same duration as a complete round-trip Mars mission, with three years being somewhat higher. In the two year case then, it may be possible to make use of consumables (and equipment) that was intended for the nominal mission in the case of the abort, although this would tend to preclude the use of prepositioned or in-situ consumables during the nominal mission. Additionally, options to include emergency rations (which can significantly reduce the mass of food), reduce consumption of water (such as through decreased washing allocations), and the conversion of propellants to consumables (oxygen and water, both of which benefit from propellants including hydrogen and oxygen in their composition), should be investigated in considering how an architecture could enable such an abort mode. Supporting the crew on this abort return may indeed be one of the most challenging aspects of employing 
these abort trajectories, particular if the 3 year free return is selected (perhaps based upon its lower Mars entry velocity.)

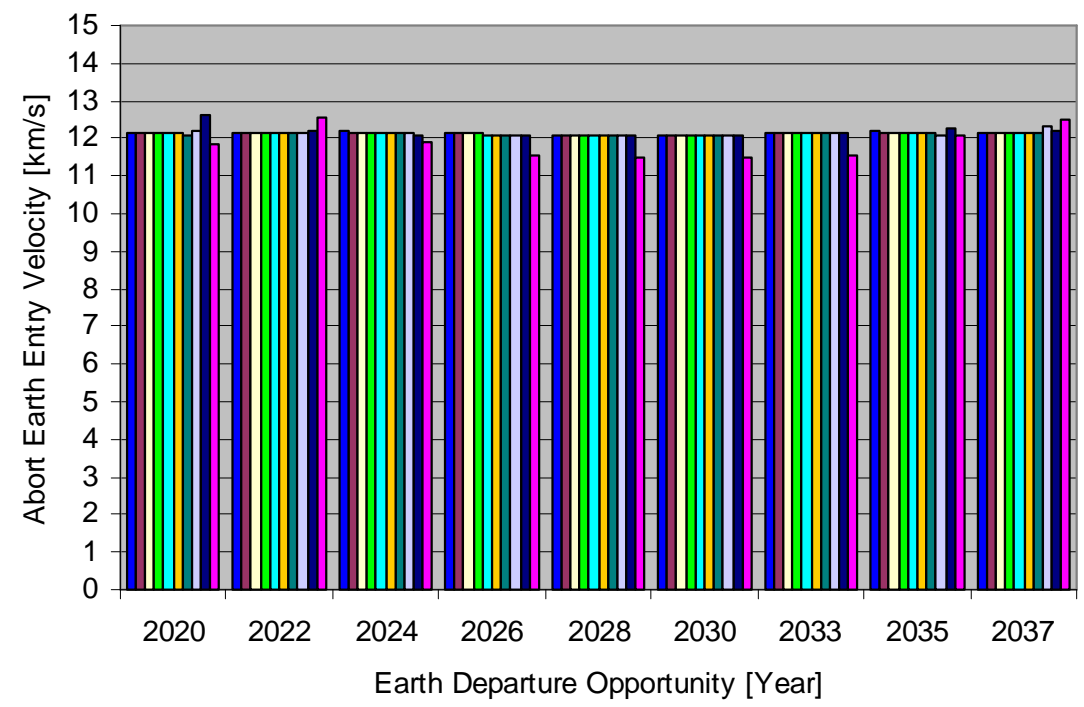

- Free Return (2 year return)

$\square$ Propulsive Abort ( $0.6 \mathrm{~km} / \mathrm{s}$ abort delta-v) $\checkmark$ Propulsive Abort (0.9 km/s abort delta-v) $\square$ Propulsive Abort (1.2 km/s abort delta-v) $\square$ Propulsive Abort (1.5 km/s abort delta-v) $\square$ Propulsive Abort (1.8 km/s abort delta- $\mathrm{v})$ 口 Propulsive Abort $(2.1 \mathrm{~km} / \mathrm{s}$ abort delta- $\mathrm{v})$ $\square$ Propulsive Abort (2.4 km/s abort delta-v) - Propulsive Abort (2.7 km/s abort delta-v) $\square$ Free Return (3 year return)

Figure 14. Earth arrival entry velocities for Earth-Mars free return and propulsive abort trajectories over Earth departure opportunities from 2020 to 2037.

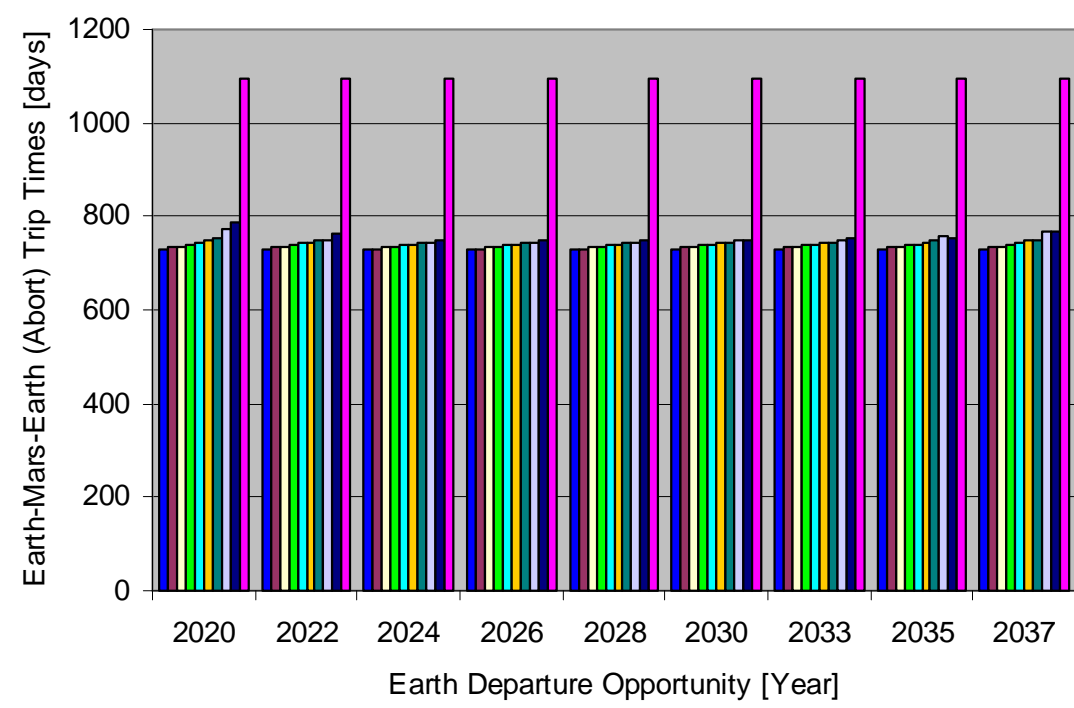

$\square$ Free Return (2 year return)

$\square$ Propulsive Abort (0.6 km/s abort delta-v) $\square$ Propulsive Abort (0.9 km/s abort delta- $\mathrm{v})$ $\square$ Propulsive Abort (1.2 km/s abort delta-v) $\square$ Propulsive Abort (1.5 km/s abort delta-v) $\square$ Propulsive Abort (1.8 km/s abort delta-v) $\square$ Propulsive Abort (2.1 km/s abort delta-v) $\square$ Propulsive Abort (2.4 km/s abort delta-v)

- Propulsive Abort (2.7 km/s abort delta-v) $\square$ Free Return (3 year return)

Figure 15. Total in-space times in case of an abort for Earth-Mars free return and propulsive abort trajectories over Earth departure opportunities from 2020 to 2037.

As in the case of the fast conjunction trajectories discussed earlier, the choice of outbound Earth-Mars trajectory will impact the stay time available at Mars. Figure 16 presents the stay times for the abort trajectories investigated (in the case where the abort is not performed and the crew stays at Mars), relative to the subsequent 270 day return transfer as in the previous section (see Figure 8 for changes resulting from other return durations). Again, this is likely not to be a major driver in trajectory selection, but can provide insight into changing exploration capability resulting from various trajectory selections. The abort trajectories for the most part have higher surface stays than the standard fast conjunction transfers, due to their even faster outbound transit times, with the exception of the 3 year free return, which in some opportunities can have significantly shorter mission durations. In the case of the propulsive abort trajectories, as the abort delta-v decreases (with the 2 year free return in the limit) the Mars stay 
time consistently increases, although the sensitivity varies with opportunity. These increases in stay time correspond with decreases in outbound transit duration.

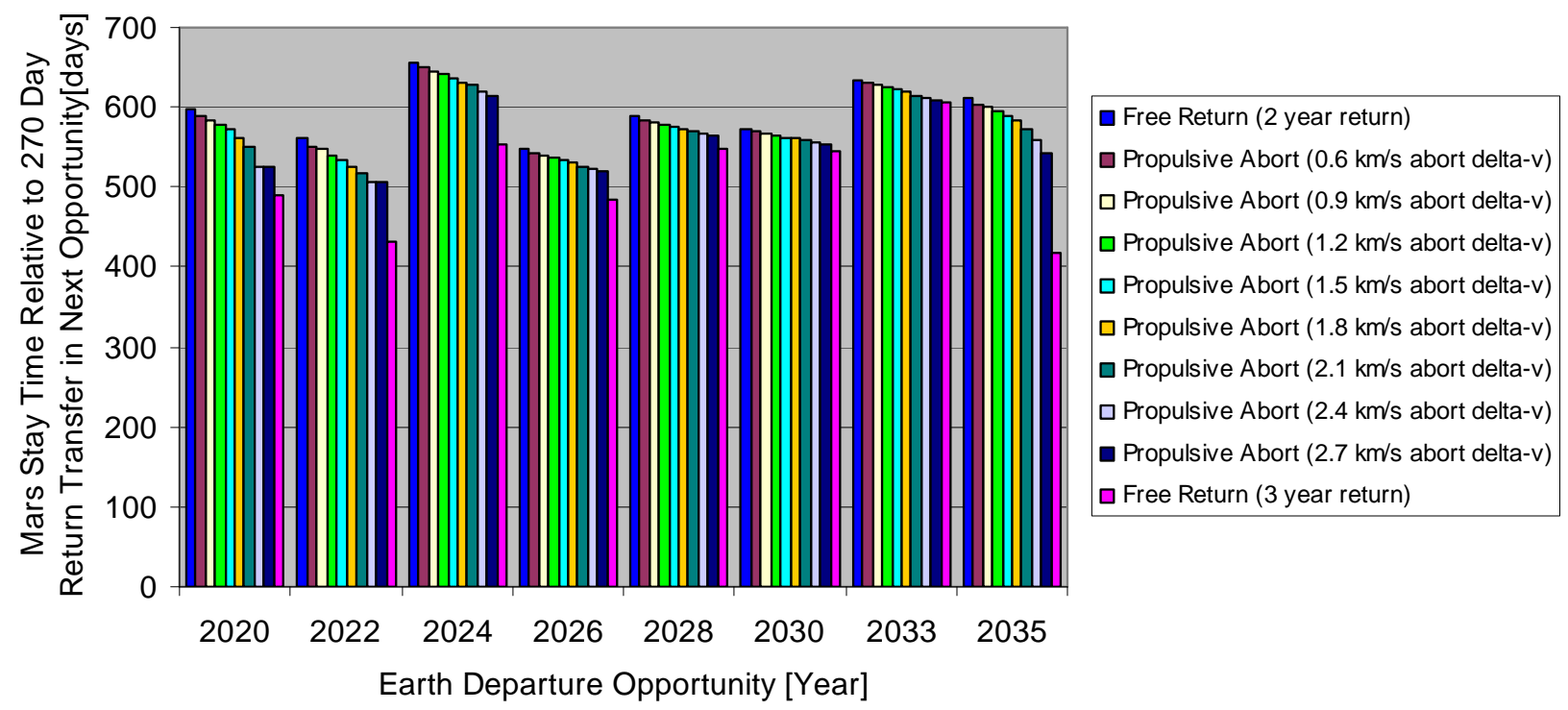

Figure 16. Mars stay times for Earth-Mars free return and propulsive abort trajectories over Earth departure opportunities from 2020 to 2035 . The stay times are computed assuming a Mars-Earth return transfer of 270 days in the immediately following Earth return opportunity (e.g., the 2022 Earth return opportunity in the case of a 2020 Earth departure).

\section{Aeroassist Maneuver Considerations for Human Mars Mission Trajectories}

Human Mars Missions utilizing aeroassist maneuver trajectories require a vehicle able to withstand the intense deceleration and aerothermal environment encountered during atmospheric entry. Uncrewed mission elements may perform aerocapture or direct entry at Mars arrival. Crewed mission elements will likely perform aerocapture at Mars arrival. An aerocapture maneuver will help crewed mission elements mitigate the large uncertainties inherent in the Mars atmosphere. In addition, aerocapture will allow time for final system check-out in orbit before descent to the surface as well as changes to landing site if required. At the end of the crew's Mars stay, they will depart for Earth. As the return vehicle nears Earth, the crew will likely transfer to a dedicated entry vehicle which will then perform direct entry at Earth. The development of practical, reliable, high performance aeroassist subsystems will demand significant technology investment. The magnitude of the required technology investment is related to the severity of the aerothermal and deceleration environment the aeroassist vehicle must pass through, which is directly related to the velocity of the vehicle at Mars or Earth arrival.

\section{A. Aeroassist at Mars Arrival}

Human Mars missions require delivery of large mass payloads to orbit or the Mars surface, with corresponding entry masses ranging from several tens of metric tonnes to perhaps over 100 metric tonnes. Large vehicles will be required to transport the large volume, large mass payloads to parking orbits and the surface, such as human habitats, ascent vehicles and return vehicles. The payload mass will drive the size of aeroshells, with 100 metric tonne entry masses perhaps requiring heatshields with forebody diameters of $15 \mathrm{~m}$ or greater. ${ }^{5,14,15,16}$ Significant technology investment in several areas is required for human exploration aeroassist maneuvers at Mars. The manufacture and qualification of large diameter heatshields presents a major engineering challenge. The large heatshields required for aeroassist maneuvers at Mars will impact the heavy life launch vehicle requirements, assuming they are launched fully assembled. Heat shield assembly in orbit also introduces a number of problems, such as qualification of a sectional heat shield assembled in low Earth orbit. In addition, vehicles performing entry at 
Mars directly from an interplanetary trajectory will experience a severe heating environment, which becomes more severe with increasing entry velocity.

An L/D of 0.3 represents an achievable value for a blunt body entry vehicle flying at a realistic angle of attack. Several blunt body shapes exist that can achieve this performance. While entry vehicles with L/Ds greater than 0.3 are conceivable for large vehicles, they often achieve a higher L/D by decreasing drag rather than by increasing lift. This can pose a severe problem at Mars, where atmospheric density is low and it is difficult to decelerate the vehicle to the proper velocity before it impacts the surface or exits the atmosphere.

Figure 17 shows the range of entry flight-path angles and velocities that provide acceptable aerocapture trajectories for a $100 \mathrm{mt}$ vehicle with a $15 \mathrm{~m}$ aeroshell generating an L/D of 0.3 , including a $5 \mathrm{~g}$ acceleration limit for crew factors reasons. In order to insure successful aerocapture, some margin must be left in entry flight-path angle to account for

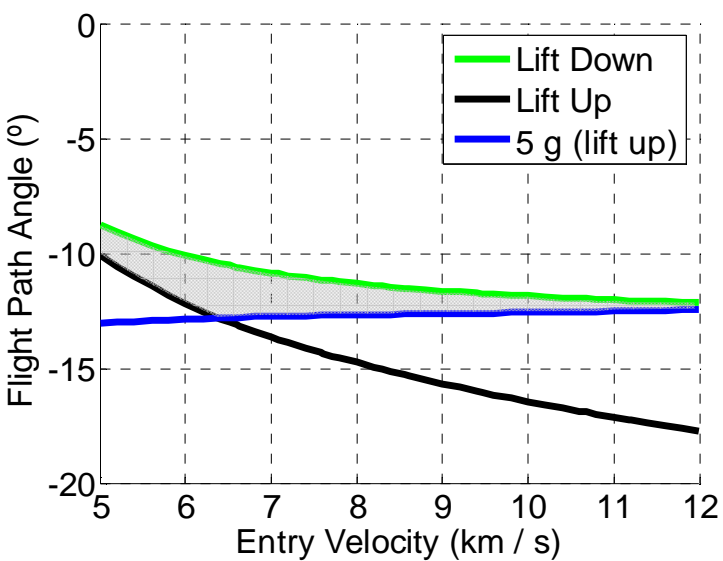

Figure 17. Aerocapture corridor width as a function of Mars entry velocity for a $100 \mathrm{mt}$ vehicle with a 15 $m$ aeroshell generating an $L / D$ of 0.3 . $^{16}$

navigation uncertainty at Mars arrival. Current robotic Mars missions have demonstrated navigation accuracies of approximately $+/-0.5$ degrees, resulting in a required entry corridor width of 1 degree ${ }^{16,17}$ Based on this limit, to maintain adequate corridor margin for aerocapture within the $5 \mathrm{~g}$ constraint, it is recommended that entry velocities be limited to approximately $9 \mathrm{~km} / \mathrm{s}$ and less. Direct entry at Mars is subject to the same navigation uncertainty as aerocapture. To maintain a corridor of $1 \mathrm{deg}$, the entry vehicle previously described must have an entry velocity of 9 $\mathrm{km} / \mathrm{s}$ or less. Direct entry at high velocity also produces an extreme heating environment with heat rates approaching $500 \mathrm{~W} / \mathrm{cm}^{2}$. In comparison, current Mars entry experience is limited to heat rates of approximately $120 \mathrm{~W} / \mathrm{cm}^{2} .{ }^{18}$ The required heating magnitude coupled with the size of the heat shield will present a significant thermal protection system (TPS) development challenge. To minimize the TPS technology investment required for direct entry, the lowest possible entry velocity and environments that maintain a low radiative heating contribution are preferred. For the purposes of conceptual-level Mars mission design we suggest a Mars entry velocity limit of $9 \mathrm{~km} / \mathrm{s}$, although more detailed analysis is required for the specific mission under development.

\section{B. Aeroassist at Earth Return}

Returning a human crew to Earth from Mars requires investment in a different set of aeroassist technologies than those required for Mars arrival. Earth entry will occur at much higher velocities $(12+\mathrm{km} / \mathrm{s})$ in a dense atmosphere. While the dense atmosphere eases $\mathrm{L} / \mathrm{D}$ requirements, the deceleration environment requires a greater $\mathrm{L} / \mathrm{D}$, and the higher entry velocity and density increase both the peak heat rate and integrated heat load significantly, which places additional stress on the TPS. The crewed earth entry vehicle function will likely be performed by an upgraded version of the Crew Exploration Vehicle (CEV) currently under development by NASA, which has a $5 \mathrm{~m}$ diameter.

Currently only two human-rated forebody TPS options are in active use: the reusable tiles used by the Space Transportation System (STS) and the Russian Soyuz single-use heatshield. Today, only STS tiles are available to NASA. The tiles were designed specifically for use with STS, a vehicle that uses a high L/D to reduce peak heat rate. Thus, STS tiles are only effective for peak heat rates below approximately $35 \mathrm{~W} / \mathrm{cm}^{2}$. In contrast, a blunt body entry vehicle returning from Mars at $12.5 \mathrm{~km} / \mathrm{s}$ may experience heat rates in excess of $400 \mathrm{~W} / \mathrm{cm}^{2}$. Direct entry at Earth at $14 \mathrm{~km} / \mathrm{s}$ produces heat rates well above $1000 \mathrm{~W} / \mathrm{cm}^{2} .{ }^{19}$ There are currently no human-rated heatshield materials available that can withstand the extreme heating environment encountered when returning from Mars. However, several promising new forebody TPS materials have recently been successfully demonstrated by the robotic exploration program and several other materials are being considered for the CEV and other planetary exploration programs. ${ }^{20}$ 
The development of the CEV for both LEO missions and lunar return missions implies the development and qualification of at least one new heatshield material. This material will be able to withstand direct entry for human lunar return, implying tolerance of entry velocities up to $11 \mathrm{~km} / \mathrm{s}$. Any forebody TPS material developed for human lunar return will likely be applicable for entry velocities up to $12.5 \mathrm{~km} / \mathrm{s}$, and should be developed with this requirement in mind. This velocity range includes the lowest Mars return velocities, and is therefore the minimum technology investment required for human return from Mars.

To facilitate fast transfer return trajectories from Mars (180 days) in all opportunities, the Earth return vehicle must be able to withstand entry at velocities up to $14.7 \mathrm{~km} / \mathrm{s}$. Entry at this velocity may produce heat rates in excess of $2000 \mathrm{~W} / \mathrm{cm}^{2}$ and integrated heat loads in excess of $100 \mathrm{~kJ} / \mathrm{cm}^{2} .{ }^{19}$ At these velocities,

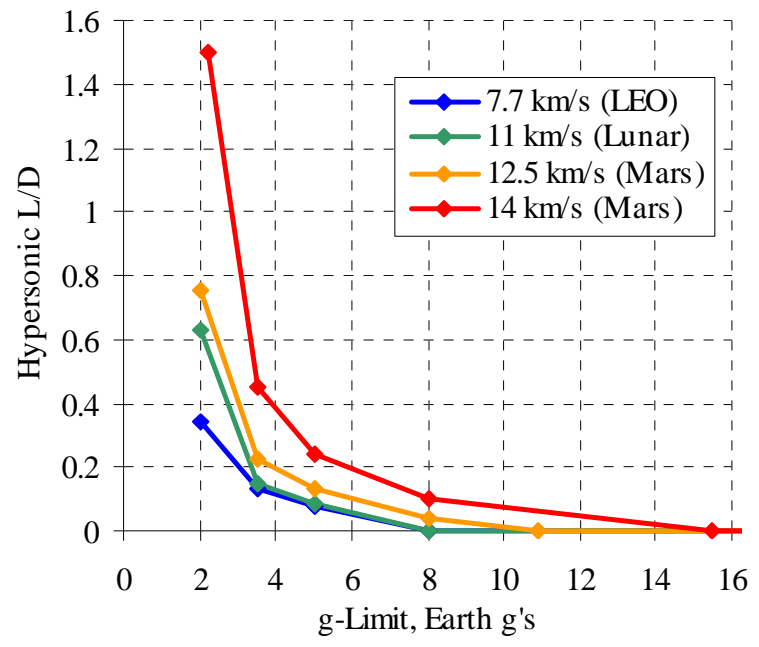

Figure 18. Required lift-to-drag ratio for direct entry with a given g-limit. ${ }^{19}$ radiative heating accounts for $50 \%$ or more of the total heat pulse, creating a heating environment that is not well understood. Developing and qualifying a heatshield that can withstand entry at $14 \mathrm{~km} / \mathrm{s}$ represents a significant engineering challenge. At velocities higher than $14 \mathrm{~km} / \mathrm{s}$, the heating environment becomes extreme as radiative heating becomes the dominant mechanism of heat transfer. At Earth entry velocities greater than $14 \mathrm{~km} / \mathrm{s}$, TPS mass fraction begins to dominate entry vehicle mass. Increased entry vehicle mass is a significant penalty to incur for additional interplanetary trajectory flexibility as the entry vehicle must be transported to Mars and back. Lastly, qualifying new TPS materials becomes more difficult as the heating environment becomes more extreme.

While TPS development and qualification for Mars return will require significant investment, NASA's current CEV moldline provides adequate aerodynamic deceleration performance. Figure 18 shows the hypersonic L/D required for a given peak deceleration at Earth. The current CEV design calls for an Apollo-style blunt body with an L/D of approximately 0.3 . Figure 18 shows that this is adequate to limit peak deceleration to $5 \mathrm{~g}$ 's even at entry velocities of $14 \mathrm{~km} / \mathrm{s}$.

The minimum technology required for human Mars return at Earth will be an extension of the systems under development for the lunar exploration program. Systems currently under development can likely accommodate Earth return speeds as high as $12.5 \mathrm{~km} / \mathrm{s}$, providing return trajectories in most opportunities for transfers of less than 210 days. To increase mission flexibility and allow fast returns for human Mars missions at all opportunities, it may be necessary to invest significant resources to develop and qualify a TPS that can withstand Earth entry at $14 \mathrm{~km} / \mathrm{s}$. This development and qualification represents a significant scientific and engineering challenge that will have significant cost and schedule requirements. To enter at velocities higher than $14 \mathrm{~km} / \mathrm{s}$, TPS development will become more difficult and a new vehicle moldline may be required to maintain a $5 \mathrm{~g}$ acceleration limit.

\section{Conclusions}

We have presented a variety of trajectory options for human Mars missions. From a propulsive perspective, in addition to standard conjunction class trajectories, 2 year free return, 3 year free return, and propulsive abort trajectory options appear feasible. In many cases, however, a trade will exist between the interplanetary trajectory chosen and its Earth or Mars entry system requirements.

Based upon the corridor width lower limit of 1 degree suggested in the analysis, and its resulting $9 \mathrm{~km} / \mathrm{s}$ upper limit on Mars entry velocity, Earth-Mars trajectories presented in Table 1 and Table 2 are feasible in the given opportunities. Using a standard conjunction trajectory, Earth-Mars transit times of 180 days or less are feasible in all opportunities other than 2030, which allows for Earth-Mars transit times of 190 days or less. The 2 year free return is only feasible within a subset of the opportunities based upon the entry corridor limit, while the three year free 
return is feasible in all opportunities. The propulsive abort can be achieved for relatively low delta- $\mathrm{v}$ in approximately half of the opportunities, with substantially higher abort delta-v's required in some opportunities.

Table 1. Standard Conjunction Trajectory Minimum Earth-Mars Transit Time by Opportunity for $9 \mathrm{~km} / \mathrm{s}$ Mars Entry Velocity Limit

\begin{tabular}{|l|c|c|c|c|c|c|c|c|c|}
\hline Opportunity & 2020 & 2022 & 2024 & 2026 & 2028 & 2030 & 2033 & 2035 & 2037 \\
\hline Minimum Transfer Time [days] & $<=180$ & $<=180$ & $<=180$ & $<=180$ & $<=180$ & $<=190$ & $<=180$ & $<=180$ & $<=180$ \\
\hline
\end{tabular}

Table 2. Free Return Trajectory Feasibility and Minimum Abort Delta-V for Propulsive Aborts by Opportunity for $9 \mathrm{~km} / \mathrm{s}$ Mars Entry Velocity Limit

\begin{tabular}{|l|c|c|c|c|c|c|c|c|c|}
\hline Opportunity & $\mathbf{2 0 2 0}$ & $\mathbf{2 0 2 2}$ & $\mathbf{2 0 2 4}$ & $\mathbf{2 0 2 6}$ & $\mathbf{2 0 2 8}$ & $\mathbf{2 0 3 0}$ & $\mathbf{2 0 3 3}$ & $\mathbf{2 0 3 5}$ & $\mathbf{2 0 3 7}$ \\
\hline 2 Year Free Return & Yes & Yes & No & No & No & No & No & No & Yes \\
\hline 3 Year Free Return & Yes & Yes & Yes & Yes & Yes & Yes & Yes & Yes & Yes \\
\hline Propulsive Abort Delta-V [km/s] & $<=0.6$ & $<=0.6$ & $<=0.6$ & $<=1.8$ & $>2.7$ & $>2.7$ & $<=2.7$ & $<=0.6$ & $<=0.6$ \\
\hline
\end{tabular}

For Earth entry, a $12.5 \mathrm{~km} / \mathrm{s}$ capability appears readily achievable, while up to $14 \mathrm{~km} / \mathrm{s}$ of entry velocity capability may be possible given additional investment. Table 3 and Table 4 show the change in Mars-Earth transfer time that can be achieved through increasing the Earth entry velocity limit. Increasing the limit to $14 \mathrm{~km} / \mathrm{s}$ can decrease transit times to under 200 days in all opportunities, as opposed to only being able to achieve transfers under 250 days in some opportunities with a $12.5 \mathrm{~km} / \mathrm{s}$ entry velocity limit.

Table 3. Standard Conjunction Trajectory Minimum Mars-Earth Transit Time by Opportunity for $12.5 \mathrm{~km} / \mathrm{s}$ Earth Entry Velocity Limit

\begin{tabular}{|l|r|r|r|r|r|r|r|r|r|}
\hline Opportunity (12.5 km/s Entry Limit) & 2020 & 2022 & 2024 & 2026 & $\mathbf{2 0 2 8}$ & $\mathbf{2 0 3 0}$ & $\mathbf{2 0 3 3}$ & $\mathbf{2 0 3 5}$ & $\mathbf{2 0 3 7}$ \\
\hline Minimum Transfer Time [days] & $<=180$ & $<=180$ & $<=210$ & $<=230$ & $<=250$ & $<=230$ & $<=180$ & $<=180$ & $<=180$ \\
\hline
\end{tabular}

Table 4. Standard Conjunction Trajectory Minimum Earth-Mars Transit Time by Opportunity for $14 \mathrm{~km} / \mathrm{s}$ Earth Entry Velocity Limit

\begin{tabular}{|l|r|r|r|r|r|r|r|r|r|}
\hline Opportunity (14 km/s Entry Limit) & $\mathbf{2 0 2 0}$ & $\mathbf{2 0 2 2}$ & $\mathbf{2 0 2 4}$ & $\mathbf{2 0 2 6}$ & $\mathbf{2 0 2 8}$ & $\mathbf{2 0 3 0}$ & $\mathbf{2 0 3 3}$ & $\mathbf{2 0 3 5}$ & $\mathbf{2 0 3 7}$ \\
\hline Minimum Transfer Time [days] & $<=180$ & $<=180$ & $<=180$ & $<=190$ & $<=200$ & $<=180$ & $<=180$ & $<=180$ & $<=180$ \\
\hline
\end{tabular}

Overall it appears that conjunction-class trajectories are quite feasible, and include options for aborting paths for the crew in some if not all opportunities (depending on the abort duration feasible.) Attention should be paid to entry velocity constraints driven by aeroassist systems in the selection and detailed design of trajectories for human Mars missions.

\section{Acknowledgments}

The analysis and results presented in this paper are based in part upon work performed as part of sub-contracts from the Charles Stark Draper Laboratory to the Massachusetts Institute of Technology and the Georgia Institute of Technology, as part of a NASA Concept Exploration and Refinement Study (NASA contract number NNT04AA10C).

\section{References}

${ }^{1}$ Walberg, G. D., "How Shall We Go to Mars? A Review of Mission Scenarios", Journal of Spacecraft and Rockets, Vol. 30, No. 2, 1993, pp. 129-139.

${ }^{2}$ Landau, D. F. and Longuski, J. K., "A Reassessment of Trajectory Options for Human Missions to Mars", AIAA 2004-5095, AIAA/AAS Astrodynamics Specialist Conference and Exhibit, Providence, RI, August 2004.

${ }^{3}$ Vallado, David A., Fundamentals of Astrodynamics and Applications, McGraw Hill, New York, 1997

${ }^{4}$ Battin, R. H., An Introduction to the Mathematics and Methods of Astrodynamics, AIAA, Inc., New York, NY, 1999

"Concept Exploration \& Refinement Study Final Report," The Charles Stark Draper Laboratory, Cambridge, MA, September 2005.

${ }^{6}$ Braun, R.D., Powell, R.W., and Hartung, L.C., "Effect of Interplanetary Trajectory Options on a Manned Mars Aerobrake Configuration," NASA TP-3019, 1990. 
${ }^{7}$ Brauer, G. L., Cornick, D. E., and Stevenson, R., "Capabilities and Applications of the Program to Optimize Simulated Trajectories (POST),” NASA CR-2770, Feb. 1977.

${ }^{8}$ Sova, G. and Divan, P., “Aerodynamics Preliminary Analysis System II, Part II - User's Manual,” NASA CR 182077, April 1991.

${ }^{9}$ Chapman, D. R., “An Approximate Analytical Method for Studying Entry into Planetary Atmospheres,” NACA TN-4276, 1958.

${ }^{10}$ Tauber, M. E. and Sutton, K., "Stagnation-Point Radiative Heating Relations for Earth and Mars Entries," Journal of Spacecraft and Rockets, Vol. 28, No. 2, 1991, pp. 40-42.

${ }^{11}$ Zubrin, R.M., D. Baker, and O. Gwynne, "Mars Direct: A Simple, Robust, and Cost- Effective Architecture for the Space Exploration Initiative", AIAA 91-0326, Aerospace Sciences Meeting, Reno, NV, January, 1991.

${ }^{12}$ Hoffman, S., Kaplan, D. (editors), The Reference Mission of the NASA Mars Exploration Study Team, NASA SP-6017, Johnson Space Center, Houston, Texas, 1997.

${ }^{13}$ Okutsu, M. and Longuski, J. K., "Mars Free Returns via Gravity Assist from Venus", Journal of Spacecraft and Rockets, Vol. 39, No. 1, 2002, pp. 31-36

${ }^{14}$ Cruz, J.R. et al., "Entry, Descent, and Landing Technology Concept Trade Study for Increasing Payload Mass to the Surface of Mars," 4th International Symposium on Atmospheric Reentry Vehicles and Systems, Arachon, France, March 2005.

${ }^{15}$ Wells, G. et al., "Entry, Descent and Landing Challenges of Human Mars Exploration," AAS 06-072, 29th AAS Guidance and Control Conference, Breckenridge, CO, February 2006.

${ }^{16}$ Braun, R.D. and Manning, R.M., "Mars Exploration Entry, Descent and Landing Challenges," IEEEAC \#0076, IEEE Aerospace Conference, Big Sky, MT, March 2006.

${ }^{17}$ Braun, R.D. and Powell, R.W., "Aerodynamic Requirements of a Manned Mars Aerobraking Transfer Vehicle," Journal of Spacecraft and Rockets, Vol. 28, No. 4, 1991, pp. 361-367.

${ }^{18}$ Milos, F.S. et al, "Mars Pathfinder Entry Temperature Data, Aerothermal Heating, and Heatshield Material Response," Journal of Spacecraft and Rockets, Vol. 36, No. 3, 1999, pp. 380-391.

${ }^{19}$ Putnam, Z. R. et al. "Entry System Options for Human Return from the Moon and Mars," AIAA 2005-5915, AIAA

Atmospheric Flight Mechanics Conference, San Francisco, CA, August 2005.

${ }^{20}$ Laub, Bernard, "Thermal Protection Concepts and Issues for Aerocapture at Titan," AIAA 2003-4954, 39th AIAA Joint Propulsion Conference and Exhibit, Huntsville, AL, July 2003. 